\title{
Improving outcomes in spinal surgery patients
}

\author{
Padmaja Durga
}

\section{INTRODUCTION}

The number of patients submitted to major spine surgery is progressively increasing. Surgery on the spine can result in debilitating complications such as neurological deficits, mechanical instability and intractable pain. Outcome is the final output measured as morbidity, mortality and quality of life. Outcome is the result of many interventions during patient course, including economic costs and the importance of an appropriate treatment. Complications are the episodes that may affect patient outcome and may require intervention, further diagnostic tests or monitoring. Outcome assessment in spinal disorders is imperative to help monitor the safety and efficacy of the treatment in an effort to change the clinical practice and improve patient outcomes.

\section{IMPROVING OUTCOMES IN SPINE SURGERY - ROLE OF ANAESTHESIOLOGIST}

Though most complications are related to surgery, the anaesthesiologist must also have knowledge of how to prevent, identify and diagnose complications of spine surgery. Good surgical and anaesthetic management protocols may improve quality of outcome. These protocols should be primarily aimed at prevention, early detection and early intervention of complications to improve outcome.

\section{Pre-anaesthetic evaluation}

Avoiding complications in spine surgery begins with careful patient selection and pre-operative evaluation. Pre-anaesthetic assessment varies depending on the approach required, the pathology involved, the

Department of Anaesthesiology and Intensive Care, Nizam's Institute of Medical Sciences, Hyderabad, Telangana, India

\begin{tabular}{|l|l|}
\hline \multicolumn{2}{|c|}{ Access this article online } \\
\hline Quick Response Code: & Website: \\
\hline & www.jnaccjournal.org \\
\cline { 2 - 2 } & \\
\hline & DOI: \\
\hline
\end{tabular}

invasiveness of the procedure and the presence of other comorbidities, which may be part of the spinal disease. Assessment also varies depending on whether the surgery is elective or emergent. A complete medical evaluation includes basic historical information such as medical problems, surgeries, current medications, allergies, substance use and prior issues with anaesthesia or surgery. Of particular importance is identifying pre-operative medical conditions that increase the risk of surgery and anaesthesia for the patient. These conditions include heart disease, diabetes, chronic obstructive pulmonary disease and bleeding dyscrasias. Pre-operative optimisation of cardiac, pulmonary, renal and nutritional status has been correlated with improved outcomes in surgical patients overall. ${ }^{[1]}$ Identification of these medical risks can then be weighed against the type and duration of surgery including number of levels, estimated blood loss and expected post-operative course.

Careful physical examination can also yield important information regarding positioning during surgery and airway issues. In particular, surgical approaches to the cervical spine often require meticulous attention to positioning and can make intubation more challenging particularly in the setting of instability. Flexion and extension manoeuvres can be performed while the patient is awake to assess for evidence of neurologic symptoms. In particular, patients with severe arthritis or complex trauma with instability may have limited neck and jaw mobility necessitating fibre optic intubation. Knowledge of these circumstances before surgery promotes safety and efficiency in the operating room.

\section{Positioning-related complications}

Spinal surgery is associated with complex morbidities when there is inattention to proper positioning technique. Problems arising from malpositioning of the patient undergoing spinal surgery are attributed to

This is an open access article distributed under the terms of the Creative Commons Attribution-NonCommercial-ShareAlike 3.0 License, which allows others to remix, tweak, and build upon the work non-commercially, as long as the author is credited and the new creations are licensed under the identical terms.

For reprints contact: reprints@medknow.com

How to cite this article: Durga P. Improving outcomes in spinal surgery patients. J Neuroanaesthesiol Crit Care 2016;3:88-96. 
body habitus and the body's contact with specialised and complex operating room table frames. Common problems associated with the prone position are brachial plexus injury, cervical spine nerve injuries and post-operative visual loss. The use of a checklist for nurse anaesthetists who provide care for patients undergoing spinal surgery will serve as a systematic aid to memory and enable the anaesthetist to adhere to proper positioning techniques in this patient population and thereby improve outcomes. ${ }^{[2]}$

\section{Neurological complications}

Quadriplegia: Extreme rotation, extension or flexion of the head can result in cervical spinal cord damage. Older patients with severe cervical spondylosis are high risk.

Prevention: Awake intubation, awake positioning, neutral or near-neutral positioning and neuromonitoring.

\section{Brachial plexus stretch injury}

Mechanism

- Prone: If the arms are abducted $>90^{\circ}$

- Supine: If the shoulders are aggressively taped down lateral decubitus: If you forget to put an axillary roll under the dependent side

- Presentations: Shoulder or supraclavicular fossa pain, mixed motor and sensory deficits

- Prognosis: Majority of the patients has spontaneous improvement within 3-6 months

- Prevention: Proper attention to positioning.

\section{Peripheral nerve compression}

\section{Eye complications}

They can occur in supine, lateral and prone positions. There is a 10 -fold increase in complications in prone position. Incidence of eye complications according to a survey is $1 \%$. Incidence of perioperative blindness is $0.05-1 \%$. Complications due to alterations in blood flow to the eyeball or optic nerve, either by decreased perfusion or by decreased embolism, are more frequent than direct pressure on the periorbital area or the globe. ${ }^{[3]}$

\section{Corneal abrasion}

Corneal abrasion is the most common surgical and general anaesthesia-related eye complication. Direct result of lagophthalmos is 'incomplete closure of the eye. Management: Most cases are self-limiting.

\section{Treatment}

\section{Ophthalmologist consult}

Topical eye antibiotics - Avoid topical eye anaesthetics: Delay the corneal epithelisation and promote keratitis.

\section{Ischaemic optic neuropathy}

Posterior ischaemic optic neuropathy is the most commonly reported visual loss secondary to prone positioning in spine surgery. Almost always irreversible visual loss. ${ }^{[4,5]}$

Risk factors: Blood loss $>4 \mathrm{~L}$, hypotensive event or relative hypotension over an extended period and long surgery.

\section{Central retinal artery occlusion}

It is the second most common cause of post-operative blindness with general anaesthesia and prone positioning, almost always irreversible visual loss. Direct or indirect pressure on the eye increases intraocular pressure. ${ }^{[6]}$

\section{Cortical blindness cortical blindness}

Occipital lobe stroke is usually bilateral. Partial to near complete recovery is expected.

Risk factors: Hypoxia - Blood loss and hypotension.

\section{SURGICAL COMPLICATIONS}

It important for the anaesthesiologist to understand the causes of surgical complication, anticipate and prepare for early identification and management of these complications to improve the outcomes.

The complications depend on the location and approach. Re-operations are associated with higher complication. ${ }^{[7]}$

\section{SURGICAL APPROACH-RELATED COMPLICATIONS}

\section{Cervical spine}

\author{
Complications of cervical spine surgery \\ Oesophageal injury \\ Vascular injury \\ Carotid sheath \\ Thyroid vessels \\ Vertebral artery \\ Neurological injury \\ Thoracic duct injury \\ Oesophageal injury: Incidence - $0.2-0.4 \%{ }^{[8]}$
}

\section{Aetiology}

Inappropriate retractor placement, excessive force during retraction or sharp retractor teeth during retraction and intraoperative trauma by a high-speed drill or sharp instrument.

Late erosion: Rare, caused due to loosening and migration of the implant. 


\section{Presentation}

Intraoperative detection: $30 \mathrm{cc}$ of indigo carmine dye through NGT - modification of above with Foley catheter proximal and distal.

Delayed presentation: Subcutaneous emphysema, dysphagia, odynophagia, neck swelling, fever, early wound infection and abscess.

\section{Prevention}

Retractor teeth under the longus colli muscles, oesophagus, should be directly protected by hand-held retractors during use of the high-speed burr.

\section{Treatment}

The treatment can be done by intraoperative thoracic or general surgeon consultation. A primary repair can be done with pedicle muscle flap (e.g., sternocleidomastoid) to protect the repair. If still in doubt, a feeding tube is placed and investigated post-operatively.

\section{Consequences}

If missed, mortality $20 \%$ if treated within $24 \mathrm{~h}$ and $50 \%$ if treatment delayed $>24 \mathrm{~h}$.

\section{Vascular injuries}

Vascular injuries in the neck not only result in bleeding but can also cause neurological injury and airway complications. ${ }^{[9]}$

\section{Carotid sheath}

Carotid artery or internal jugular vein injury are rare, result from sharp retractor teeth or during dissection.

Management: Artery - primary repair; vein - primary repair or ligation.

\section{Thyroid vessels}

Bleeding from superior (above $\mathrm{C} 4$ ) and inferior (below C6) thyroid arteries can be controlled easily. However, the close proximity of superior laryngeal nerve with superior thyroid artery and recurrent laryngeal nerve with inferior thyroid artery must be kept in mind.

\section{Vertebral artery}

- Incidence: 0.3\% (of 1976 patients) anterior approach

- Anatomy:The mean distance from the uncovertebral joint to the transverse foramen: $5.5 \mathrm{~mm}$ in the sub-axial vertebrae. The incidence of anomalies is $2.7 \%$. There can be unilateral artery displacement, with transverse foramen enlargement as far medial as the mid-vertebral body level.

\section{Management of vertebral artery injury}

- May need blood transfusion

- Surgical management

- Direct tamponade: Temporary

- Direct repair - proximal and distal ligation
- Endovascular stent or coagulation

- Repair of the vertebral artery injury is the preferred approach.

\section{Consequences of vertebral artery injury}

The predicted incidence of brainstem infarction in the presence of normal contralateral vessel is $3.1 \%$ when the left vertebral artery is ligated and $18 \%$ when the right vertebral artery is ligated.

\section{Prevention of vertebral artery injuries}

It is important to review the position of vertebral artery in pre-operative imaging, orient to midline, limit dissection to $15 \mathrm{~mm}$ from midline over $\mathrm{C} 1 \mathrm{arch}$.

\section{Neurological injuries}

Dural tear

- Incidence - 3.7\% anterior approach

- Risk factors for dural tear: Revision surgery, ossification of the posterior longitudinal ligament

- Consequences: Persistent leak, fistula formation and airway compromise

- Management: Watertight closure if feasible, fibrin glue, lumbar drain, upright position post-operatively.

Spinal cord injury

- Incidence: Anterior: 0.2-0.9\%

- Risk factors: Myelopathy, cervical kyphosis, spinal cord atrophy, spinal instability, fractures through long fused spinal segments.

\section{Mechanism of spinal cord injury}

- Problems related to positioning and/or intubation

- Direct mechanical injury: Surgical instruments, penetration of posterior cortex with drill or screws, uncontrolled intraoperative distraction (especially trauma patient with torn soft tissue), inserting the graft too far (anteroposterior diameter of graft should be $\leq 13 \mathrm{~mm}$ ), removal of osteophytes at the posterior vertebral body margin, epidural haematoma.

\section{Prevention of spinal cord injury}

- Maintenance of systolic blood pressure $>80 \mathrm{mmHg}$

- Avoidance of excessive extension or distraction (consider pre-intubation positioning and neurological examination)

- Neuromonitoring

- Steroids.

\section{Neuromonitoring}

Paraparesis, paraplegia and quadriplegia are complications of spinal surgery. Intraoperative monitoring (IOM) of neural function is used to warn of the risk of surgical complications. ${ }^{[10]}$

The sensitivity and specificity of electrophysiological monitoring varied due to different warning criteria being used. The sensitivity and specificity of unimodal 
somatosensory evoked potential (SSEP) monitoring ranged from $0-100 \%$ to $27-100 \%$, respectively. The positive predictive value was $15-100 \%$, and the negative predictive value was $95-100 \% .{ }^{[10]}$ However, when SSEP monitoring is used in combination with other neurophysiologic modalities its accuracy is improved. In a meta-analysis, it was seen that both the sensitivity and the specificity of motor evoked potential (MEP) monitoring varied from $81 \%$ to $100 \%$. The positive predictive value and the negative predictive value ranged from $17-96 \%$ to $97-100 \%$, respectively. When MEP monitoring is used in combination with other neurophysiologic modalities, the overall strength with respect to sensitivity and specificity is 'high.'[10] Intraoperative electromyography (EMG) activation had a sensitivity of $100 \%$ and a specificity of $23.7 \%$ for the

\section{Table 1: Improving outcomes in spine surgery-role of anaesthesiologist}

\begin{tabular}{l} 
Through pre-operative evaluation and optimisation \\
Prevention of position related complications \\
Understanding, anticipation, identification and early \\
intervention of surgical complications \\
Neurological \\
Bleeding \\
Pain \\
Prophylaxis and prevention of wound and respiratory \\
infections \\
DVT prophylaxis \\
\hline DVT=Deep venous thrombosis \\
Table 2: Complications of positioning \\
\hline Neurological: Quadriplegia and peripheral nerve palsies \\
Eye complications \\
Excessive bleeding
\end{tabular}

detection of a new post-operative neurological deficit, whereas SSEP had a sensitivity of $28.6 \%$ and specificity of $94.7 \%{ }^{[11]}$ The combination of intraoperative EMG and SSEP monitoring is helpful for predicting and possibly preventing neurological injury during thoracolumbar spine surgery. The data on post-operative outcomes in patients who underwent pedicle screw stimulation or triggered EMG monitoring are conflicting in the literature. Multimodality IOM combines SSEPs, MEPs and EMG according to the structures at risk in surgery, taking advantage of the individual strength of each modality for accurate monitoring. ${ }^{[12]}$

\section{What to do, if there was an alert?}

Anaesthesiologists and surgeons can intervene in a variety of ways when IOM raises warnings to attempt to reduce the risk of adverse neurologic outcomes. They can modify surgery by interventions such as reducing the degree of distraction, adjusting retractors, removing or adjusting grafts or hardware, reimplanting or unclamping arteries, placing vascular bypass grafts, minimising the remaining portion of the surgery or other actions. Surgeons also have the opportunity to check a wake-up test in some patients. Adverse IOM changes predict increased risk of adverse clinical outcomes consistently. ${ }^{[13]}$

If no neuromonitoring used, deficit is usually detected in recovery room: Urgent $X$-ray to exclude dislodgement of graft or hardware, urgent magnetic resonance imaging ( \pm computed tomography) to exclude epidural haematoma and hardware malposition. If no structural problems, treatment is largely expectant.

Role of steroids in prevention and management of intraoperative spinal cord injury

Controversy exists regarding steroid use in traumatic spinal cord injury. The National Acute Spinal Cord Injury Studies II and III suggest that high-dose therapy

Table 3: Peripheral nerve injury during positioning

\begin{tabular}{|c|c|c|c|}
\hline Nerve & Mechanism & Presentation & Prevention \\
\hline Ulnar nerve & Compressed around the elbow & $\begin{array}{l}\text { Symptoms appear } \\
1 \text {-4 days post-operative, } \\
\text { resolve in weeks }\end{array}$ & $\begin{array}{l}\text { Pad under } \\
\text { the slightly } \\
\text { extended elbow }\end{array}$ \\
\hline Radial nerve & Injured if the arm hangs at the edge of the table & $\begin{array}{l}\text { Symptoms appear } \\
1-4 \text { days post-operative, } \\
\text { resolve in weeks }\end{array}$ & $\begin{array}{l}\text { Padding under } \\
\text { the arm }\end{array}$ \\
\hline $\begin{array}{l}\text { Common } \\
\text { peroneal nerve }\end{array}$ & $\begin{array}{l}\text { Compression around the fibular head } \\
\text { May occur in supine, prone or lateral position }\end{array}$ & Foot drop & \\
\hline $\begin{array}{l}\text { Lateral femoral } \\
\text { cutaneous } \\
\text { nerve injury }\end{array}$ & $\begin{array}{l}\text { Occurs in supine and prone } \\
\text { Prone: ASIS compression in the prone position } \\
\text { Supine: During bone graft harvesting at the } \\
\text { anterior iliac crest } \\
\text { At the retroperitoneum by haematoma or traction }\end{array}$ & Meralgia paraesthetica & $\begin{array}{l}\text { Avoid } \\
\text { compression } \\
\text { and retraction }\end{array}$ \\
\hline
\end{tabular}

ASIS=Anterior superior iliac spine 
Table 4: Causes of visual disturbance

\begin{tabular}{l} 
Ophthalmic \\
Corneal injury \\
ION \\
Retinal vessel occlusion \\
Non-ophthalmic \\
Cortical blindness \\
ION=Ischaemic optic neuropathy \\
Table 5: Prevention of eye complications \\
\hline A slight reverse Trendelenburg (head up) position to \\
decrease facial oedema and periorbital swelling \\
Avoid direct pressure on the eyes \\
Tape the eyes \\
Maintenance of blood pressure \\
Catching up with bleeding \\
Staging long procedure
\end{tabular}

Table 6: Causes of surgical complications

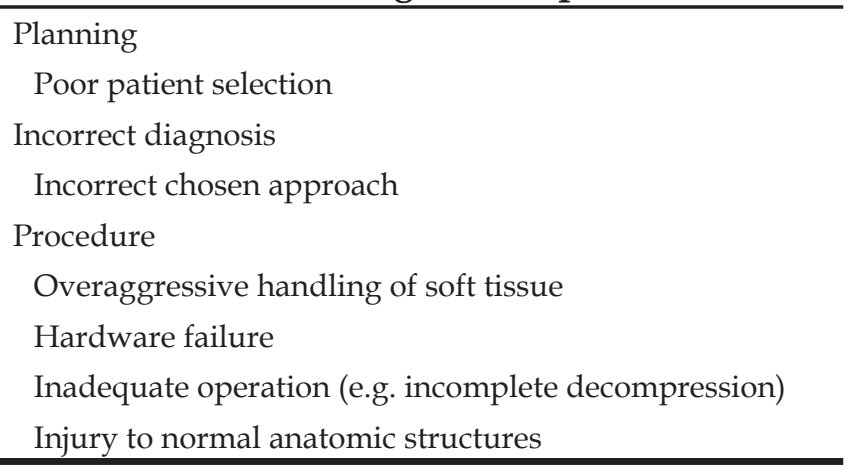

Table 7: Timing

Intraoperative
Early post-operative (within 1 week)
Late post-operative (1-6 weeks)

administered within $8 \mathrm{~h}$ of initial injury does confer a benefit in the setting of incomplete spinal cord injury. ${ }^{[14]}$ The American College of Surgeons no longer mandates its use. ${ }^{[15,16]}$ In the setting of routine, elective spine surgery, low doses (5-10 mg) of Decadron (dexamethasone) are typically given before cases involving large disc herniations, spinal cord tumours and severe canal stenosis. Such perioperative treatment is thought to preserve neural structures from inflammation and swelling post-operatively. Steroids are usually given for no longer than $24-48 \mathrm{~h}$ to avoid known complications associated with long-term steroid therapy.
Table 8: Mechanisms of vertebral artery injury

Anterior spine

Excessively wide corpectomy (limit to 15-17 mm)

Loss of the vertebral midline orientation, leading to an off- centre or oblique corpectomy

Unrecognised vertebral artery tortuosity or other anomalies, e.g., a vertebral artery located anterior to the transverse process

Using a burr to decompress the nerve root at the uncovertebral region can bind dense fibrous bands within the transverse foramen

Excessive dissection beneath the longus colli (when the artery is unprotected) between the transverse foramen

Soft lateral bone resulting from infection or tumour

Posterior spine

C1-C2 transarticular screw

C1, C2 lateral mass screws: Too medial

Over dissection lateral to the lateral masses

Table 9: Neurological injuries during cervical spine surgery

Dural tear

Spinal cord injury

C5 radiculopathy

Recurrent and superior laryngeal nerve injury

Sympathetic trunk injury

Table 10: Risk factors for post-operative acute airway compromise

\begin{tabular}{ll}
\hline Haematoma formation & Obesity \\
CSF leakage & Obstructive sleep apnea \\
Hardware or bone graft & History of asthma \\
displacement & Surgical time $>5 \mathrm{~h}$ \\
Laryngeal or prevertebral & Revision surgery \\
soft-tissue swelling & Exposure of three or \\
& more disk levels \\
& Transfusion of more \\
& than four units of blood \\
\hline
\end{tabular}

CSF $=$ Cerebrospinal fluid

Table 11: Complications of iliac donor site

\begin{tabular}{ll}
\hline Donor site pain & $\begin{array}{l}\text { Infection } \\
\text { Neurovascular injury }\end{array}$ \\
Avulsion fractures of the ASIS & abdominal contents \\
Instability of the sacroiliac joint & Cosmetic deformity \\
Haematoma & Ureteral injury \\
\hline
\end{tabular}

ASIS=Anterior superior iliac spine 


\section{C5 radiculopathy post-cervical}

\section{decompression}

C5 roots are shorter than other cervical nerve roots. C5 is usually at the midpoint of the decompressed segment and subject to the greatest stretch with shifting of the spinal cord. Restoration of the cervical lordosis shifts the spinal cord posteriorly and increases the stretch on C5 roots. Deltoid has uni segmental innervation which makes C5 palsy more clinically obvious. Prognosis is good, self-limiting but take months to recover.

\section{Recurrent laryngeal nerve}

- Incidence: $5-16 \%$

- Risk factors: Direct surgical trauma: Stretching, post-operative oedema, attempted control of inferior thyroid bleeding, revision surgery, surgery at $\mathrm{C6}$ - $\mathrm{T} 1$ region

- Clinical presentation: Hoarseness, aspiration, persistent cough, dysphagia

- Management: Spontaneous recovery is expected in most patients, but can take up to 1 year. In patients with significant aspiration, vocal cord medialisation (injecting absorbable gelatin sponge into the injured vocal cord) provides 4-6 weeks of medialisation

- Prevention: Respect the relation of superior thyroid artery (with superior laryngeal nerve) and inferior thyroid artery (with recurrent laryngeal nerve). Monitoring endotracheal tube cuff pressure and deflating the endotracheal tube cuff after placement of the retractor: Reduced the rate of injury from $6.4 \%$ to $1.7 \%$ (Apfelbaum et al. Spine 2000)

- For revisionanteriorcervicalcases:Getlaryngoscopic examination - If both recurrent laryngeal nerves are intact go to the contralateral side (to avoid scar from previous surgery) - If one of the recurrent laryngeal nerves is injured go to the ipsilateral side (side of injured nerve).

\section{Superior laryngeal nerve injury}

\section{Clinical presentations}

- Injury to sensory branch (internal laryngeal): Post-swallowing cough, choking sensation and aspiration because of loss of sensation above the vocal cords and loss of reflexive closure of vocal cords to prevent aspiration

- Injury to motor branch (external laryngeal)

- Unilateral injury: Subtle change in the pitch of voice is unnoticed, except for singers

- Bilateral injury: Voice hoarseness and tires easily.

Sympathetic trunk injury (Horner's syndrome)

- Incidence: 0.2-4\% more in revision surgery. Result from injury to the chain cephalad to the inferior half of stellate ganglion or from post-ganglionic injury

- Prevention: Dissection beneath longus colli and avoiding excessive retraction of the muscle.

\section{Thoracic duct injury}

At risk in the left-sided anterior approaches to lower cervical and cervicothoracic junction.

- Consequences: Chylo mediastinum or a chylous pleural effusion

- Management of thoracic duct injury: If detected intraoperatively, double ligation of the thoracic duct. Non-surgical management (if detected post-operatively) - reduction of the chyle flow by eliminating enteral feeds, very low fat total parenteral nutrition, drainage of pleural cavity

- Nutritional support - Prevention of sepsis: Risk of bacterial and fungal sepsis and 50\% mortality. If non-surgical treatment failed vascular clip and fibrin glue through thoracotomy or thoracoscopy.

\section{Early post-operative}

Acute airway compromise: Incidence of reintubation following anterior cervical spine surgery: $1.7-2.8 \%{ }^{[17]}$

Risk factors: The most frequent cause is laryngeal or pre-vertebral soft-tissue swelling. Peak swelling occurs on POD 2 and 3. Swelling at the C2-C4 levels was more clinically significant than it was below $\mathrm{C} 5$.

Management:

- Consider keeping patient intubated for high-risk patients

- Assess weaning parameters

- Elevation of the head of the bed

- Diuresis

- Inhaled or intravenous steroids for soft-tissue oedema.

\section{Post-operative wound haematoma}

- Incidence: $0.2-1.9 \%$

- Mechanism: Due to venous bleeding or from an unrecognised or inadequately controlled arterial source. Can occur despite placement of a post-operative drain and adequate haemostasis at the time of wound closure due to increased blood pressure, coughing, vomiting, coagulopathy and the use of an anticoagulant

- Consequences: Can cause life-threatening airway compromise, persistent wound drainage and infection. Patients with life-threatening airway compromise and apparent swelling at the site of incision are candidates for urgent wound incision and drainage at the bedside or in the operating room. 


\section{Post-operative wound infection}

Prevention of wound infection requires a joint effort from surgeon and anaesthesiologist. The rate of infection from spine surgery has been reported between $0.3 \%$ and $9 \%$ (higher than the quoted rates from the orthopaedic literature and non-contaminated procedures $-0.2 \%$ to $1.6 \%$ ). It is relatively rare in the anterior spine except after oesophageal injury or immunocompromised patients. Inadequate blood glucose control in diabetics is one of the leading risk factors for post-operative wound infection. The other risk factors include age $>60$ years, obesity, smoking and malnutrition. Intraoperative factors including increased operative time, increased blood loss and multiple staging of spinal procedures can also raise the risk of post-operative wound infection by nearly 3 -fold. A single perioperative dose of the first-generation cephalosporin is often adequate in reducing post-operative infection. In 2007, guidelines published by the North American Spine Society cite level B evidence supporting the use of perioperative antibiotics in spine surgery. Intraoperative sterile technique, judicious use of antibiotics and wound irrigation (a minimum of $2 \mathrm{~L}$ of saline irrigation in the surgical wound before closure with antibiotic such as bacitracin) proper blood glucose control, early mobilisation after surgery and adequate wound care are all essential factors in preventing operative site infections.

\section{Thoracic, lumbar and sacral approaches}

Approaches to the thoracic and lumbosacral spine share similar risks described for the cervical spine but also present their own unique challenges. Ventral approaches to the thoracic spine require, by design, thoracotomy and chest tube placement. Post-operatively, these patients often suffer from increased pain, decreased mobility and increased rates of pneumonia and atelectasis. At the thoracolumbar junction, take down of the diaphragm for ventral exposure often carries the highest morbidity. Overall, the morbidity rates for these approaches remain low at approximately $1-4 \%$ and include pneumonia, pleural tear, infection, bowel obstruction and neurologic dysfunction. Risk of injury to the thoracic spinal cord remains low at approximately $1 \%$ according to some studies. Such risk can be reduced by maintaining adequate spinal cord perfusion, both intraoperatively and post-operatively. Mean arterial blood pressure goals are usually kept in the $80-90 \mathrm{mmHg}$ range in such settings. Ventral and dorsal approaches to the lumbar and sacral spine carry less risk to neural structures as the canal widens and the spinal cord ends typically above the level of L2.

Large-vessel injury remains the most common serious injury from ventral approaches with incidence rates reported between $8 \%$ and $12 \%$. Such injury is most often secondary to venous laceration usually resulting from retraction injury.
Although rare, vascular injuries have been reported from dorsal approaches and carry a mortality rate as high as $10-40 \%$ depending on the type and location of the injury. The artery of Adamkiewicz is a large artery supplying the thoracolumbar spinal cord. It typically exits the aorta on the left side at lower thoracic or upper lumbar levels. Injury to this vessel can result in an extensive spinal cord infarction. Pre-operative angiography is often used to identify the anatomic location of this vessel to avoid injury during surgery.

Visceral injury is rare in ventral approaches since the exposure is carried out in a retroperitoneal fashion although post-operative ileus is present frequently. Injury to the lumbar sympathetic plexus occurs in about $6 \%$ of all ventral approaches to the lumbosacral spine and usually results from retraction injury. Patients typically report an ipsilateral, warm leg after surgery that often resolves spontaneously. Injury to the superior hypogastric plexus is often a troubling complication after surgery and can result in bladder dysfunction in female patients and retrograde ejaculation/sterility in male patients. Careful surgical technique and avoidance of electrocautery in the region of the pre-vertebral fascia are the best ways to avoid such complications. ${ }^{[18-20]}$

\section{Iliac graft donor site complications}

It is important to know whether autologous bone grating is intended and know its complications. ${ }^{[21]}$

\section{Donor site pain}

Acute post-operative pain and nerve injuries at anterior iliac crest bone graft can lead to neuropathic, chronic pain. ${ }^{[22]}$

- Incidence: $2.8-25 \%$

- Mechanism: Unclear; muscular or periosteal secondary to the stripping of the abductors from the ilium. May be related to injury of the superior gluteal nerve. Injury to lateral cutaneous nerve of thigh can result in meralgia paraesthetica. ${ }^{[22]}$

A small study evaluated the efficacy of pre-operative placement of transversus abdominis plane (TAP) block under ultrasound-guided technology. The authors considered TAP to be an appropriate technique for post-operative analgesia at the bone harvest as about $80 \%$ of patients had no pain at 18 months. ${ }^{[23]}$ Whatever the approach, a plan for pain relief must be detailed as the discomfort experience from the hip is significantly greater than that felt at the spine surgery. Other techniques include local infiltration, patient-controlled analgesia and opioid or steroidal injections.

Vascular injuries of graft harvesting: Harvesting iliac bone too close to the greater sciatic notch may injure superior gluteal artery and anteriorly fourth lumbar 
artery, iliolumbar artery and deep circumflex iliac artery extensive anastomoses in the pelvis overlying the iliacus muscle may be injured. They may cause brisk bleeding up to $1.5 \mathrm{~L}$.

\section{DEEP VENOUS THROMBOSIS AND PULMONARY EMBOLISM}

\section{Incidence and treatment of deep venous thrombosis in elective spine surgery}

The method of surveillance and the use or non-use of prophylaxis would influence the incidence. ${ }^{[24]}$ The only studies evaluating spine surgery cohorts without the use of mechanical or pharmacologic prophylaxis have come from Eastern Asia. The efficacy and safety of chemical prophylaxis to prevent the development of deep venous thrombosis or pulmonary embolism following spine surgery are controversial because of the possibility of epidural haematoma formation. Pre-operative risk factors identified were pre-operative walking disability and age. ${ }^{[25]}$ The overall rate of venous thromboembolism (VTE) was $1.37 \%$ (95\% confidence interval: 1.33-1.41), but varied widely depending on diagnosis $-1.03 \%$ for structural degenerative diagnoses to $10.7 \%$ for spine infection. Posterior cervical fusion had a higher rate of VTE than anterior cervical fusion, while anterior thoracolumbar and lumbosacral fusions had higher rates than the respective posterior approaches. Additional risk factors included patients receiving long spine fusions and having multiple procedures during the hospitalisation. ${ }^{[24]}$ Consideration of patient-related VTE risks, procedure-related VTE risks and the risk of neurological compromise from bleeding complications will more appropriately balance safety and effectiveness when choosing a VTE prophylaxis method. ${ }^{[26]}$ An aggressive protocol for early VTE prophylaxis after spine surgery decreases VTE incidence without increasing morbidity. ${ }^{[27]}$

\section{Minimally invasive approach}

Minimising surgical extent, operative time and blood loss can potentially reduce post-operative complications. Technological improvements in spinal instruments facilitate shorter and safer surgeries. ${ }^{[28]}$

\section{CONCLUSION}

There is no such thing as a simple spine operation. It is easier to stay out of trouble than to get out of trouble. The time expended in avoiding complications will be more than compensated by the time saved in not having to treat them. The patient's well-being is paramount. Avoiding complications in spine surgery is a complex endeavour for the spine surgeon and anaesthesiologist alike. The anaesthesiologist managing spine surgery should never hesitate to request consultation and discussion with surgeon pre-operatively and during surgery. Anaesthesiologist plays an important role in the perioperative period in improving outcome of surgery. Knowledge of proper positioning technique and awareness of the risks inherent in each surgical procedure allows both the anaesthesia and surgical teams to anticipate potential complications and improve outcomes in patients undergoing spine surgery.

\section{Financial support and sponsorship} Nil.

\section{Conflicts of interest}

There are no conflicts of interest.

\section{REFERENCES}

1. Halpin RJ, Sugrue PA, Gould RW, Kallas PG, Schafer MF, Ondra SL, et al. Standardizing care for high-risk patients in spine surgery: The northwestern high-risk spine protocol. Spine (Phila Pa 1976) 2010;35:2232-8.

2. Salkind EM. A novel approach to improving the safety of patients undergoing lumbar laminectomy. AANA J 2013;81:389-93.

3. Stambough JL, Dolan D, Werner R, Godfrey E. Ophthalmologic complications associated with prone positioning in spine surgery. J Am Acad Orthop Surg 2007;15:156-65.

4. Pierce V, Kendrick P. Ischemic optic neuropathy after spine surgery. AANA J 2010;78:141-5.

5. Kempen PM. Perioperative ischemic optic neuropathy and spine surgery: Are we asking the right questions? Anesthesiology 2012;117:431-2.

6. Sodhi HB, Salunke P, Kaur S, Singh R. Missing the 'window' might shut the light forever: Central retinal artery occlusion following spine surgery. Surg Neurol Int 2015;6:86.

7. Daniels AH, Riew KD, Yoo JU, Ching A, Birchard KR, Kranenburg AJ, et al. Adverse events associated with anterior cervical spine surgery. J Am Acad Orthop Surg 2008;16:729-38.

8. Patel NP, Wolcott WP, Johnson JP, Cambron H, Lewin M, McBride D, et al. Esophageal injury associated with anterior cervical spine surgery. Surg Neurol 2008;69:20-4.

9. Wakao N, Takeuchi M, Nishimura M, Riew KD, Kamiya M, Hirasawa A, et al. Risks for vascular injury during anterior cervical spine surgery: Prevalence of a medial loop of vertebral artery and internal carotid artery. Spine (Phila Pa 1976) 2015.

10. Fehlings MG, Brodke DS, Norvell DC, Dettori JR. The evidence for intraoperative neurophysiological monitoring in spine surgery: Does it make a difference? Spine (Phila Pa 1976) 2010;35 9 Suppl: S37-46.

11. Gunnarsson T, Krassioukov AV, Sarjeant R, Fehlings MG. Real-time continuous intraoperative electromyographic and somatosensory evoked potential recordings in spinal surgery: Correlation of clinical and electrophysiologic findings in a prospective, consecutive series of 213 cases. Spine (Phila Pa 1976) 2004;29:677-84.

12. Malhotra NR, Shaffrey CI. Intraoperative electrophysiological monitoring in spine surgery. Spine (Phila Pa 1976) 2010;35:2167-79.

13. Eager M, Shimer A, Jahangiri FR, Shen F, Arlet V. Intraoperative neurophysiological monitoring (IONM): Lessons learned from 32 case events in 2069 spine cases. Am J Electroneurodiagnostic Technol 2011;51:247-63.

14. Bracken MB, Shepard MJ, Holford TR, Leo-Summers L, Aldrich EF, Fazl M, et al.Administration of methylprednisolone 
for 24 or 48 hours or tirilazad mesylate for 48 hours in the treatment of acute spinal cord injury. Results of the third national acute spinal cord injury randomized controlled trial. National acute spinal cord injury study. JAMA 1997;277:1597-604.

15. Frampton AE, Eynon CA. High dose methylprednisolone in the immediate management of acute, blunt spinal cord injury: What is the current practice in emergency departments, spinal units, and neurosurgical units in the UK? Emerg Med J 2006;23:550-3.

16. Short DJ, El Masry WS, Jones PW. High dose methylprednisolone in the management of acute spinal cord injury - A systematic review from a clinical perspective. Spinal Cord 2000;38:273-86.

17. Sagi HC, Beutler W, Carroll E, Connolly PJ. Airway complications associated with surgery on the anterior cervical spine. Spine (Phila Pa 1976) 2002;27:949-53.

18. Sansalone CV, Soldano S, Poli C, Tripepi M, D'Aliberti GA, Rossetti O. Anterior approach to the spine. Role of the general surgeon, techniques and surgical complications. The 10-year experience of the Niguarda Hospitals. J Neurosurg Sci 2011;55:357-63.

19. Fu ZQ, Zhang ZM, Jin DD, Chen JT, Qu DB. Complications of the anterior surgical approach for thoracolumbar spine tuberculosis: Causes and countermeasures. Nan Fang Yi Ke Da Xue Xue Bao 2009;29:1229-31.

20. Cheung KM, Al Ghazi S. Approach-related complications of open versus thoracoscopic anterior exposures of the thoracic spine. J Orthop Surg (Hong Kong) 2008;16:343-7.

21. Pitzen T, Kränzlein K, Steudel WI, Strowitzki M. Complaints and findings at the iliac crest donor site following anterior cervical fusion. Zentralbl Neurochir 2004;65:7-12.

22. Yanow JH, Lorenzo LD, Worosilo SC, Pappagallo M. Successful treatment of chronic donor site pain. Anesth Pain Med 2015;5:e18777.

23. Chiono J, Bernard N, Bringuier S, Biboulet $\mathrm{P}$, Choquet $\mathrm{O}$, Morau $\mathrm{D}$, et al. The ultrasound-guided transversus abdominis plane block for anterior iliac crest bone graft postoperative pain relief: A prospective descriptive study. Reg Anesth Pain Med 2010;35:520-4.

24. Schairer WW, Pedtke AC, Hu SS. Venous thromboembolism after spine surgery. Spine (Phila Pa 1976) 2014.

25. Tominaga H, Setoguchi T, Tanabe F, Kawamura I, Tsuneyoshi Y, Kawabata N, et al. Risk factors for venous thromboembolism after spine surgery. Medicine (Baltimore) 2015;94:e466.

26. Eskildsen SM, Moll S, Lim MR. An algorithmic approach to venous thromboembolism prophylaxis in spine surgery. J Spinal Disord Tech 2015;28:275-81.

27. Cox JB, Weaver KJ, Neal DW, Jacob RP, Hoh DJ. Decreased incidence of venous thromboembolism after spine surgery with early multimodal prophylaxis: Clinical article.J Neurosurg Spine 2014;21:677-84.

28. Banczerowski P, Czigléczki G, Papp Z, Veres R, Rappaport HZ, Vajda J. Minimally invasive spine surgery: Systematic review. Neurosurg Rev 2015;38:11-26. 